\title{
Recovery of quality of life following curative intent surgery for colorectal cancer: results from the colorectal well-being (CREW) study
}

Sally Wheelwright ${ }^{1 *}$, Mubarak Patel ${ }^{1}$, Lynn Calman ${ }^{1}$, Amy Din $^{1}$, Samantha Sodergren ${ }^{1}$, Deborah Fenlon ${ }^{2}$, Janis Baird ${ }^{3}$, Jessica Corner ${ }^{4}$, Alison Richardson ${ }^{1,5}$, Peter W Smith ${ }^{6}$, Jane Winter ${ }^{5}$, Members of the Study Advisory Committee, Claire Foster ${ }^{1}$

${ }^{1}$ Health Sciences, University of Southampton, ${ }^{2}$ Human \& Health Sciences, Swansea University, ${ }^{3}$ Medicine, University of Southampton, ${ }^{4}$ Executive Office, University of Nottingham, ${ }^{5}$ University Hospital Southampton NHS Foundation Trust, ${ }^{6}$ Social Sciences, University of Southampton

\section{Introduction}

Survivors of colorectal cancer (CRC) form the largest group of cancer survivors affecting men and women but while survival rates continue to improve, knowledge about recovery of health and well-being is limited. This is partly because most previous studies are cross-sectional or did not include a pretreatment assessment ${ }^{1}$. Understanding how people recover can inform provision of care and help to prepare patients for what to expect. The ColoREctal Wellbeing (CREW) study ${ }^{2}$ was established to explore the recovery of health and well-being following curative surgery for colorectal cancer. The objectives for this analysis were i) to investigate the recovery of quality of life (QOL) over 5 years following curative intent treatment of colorectal cancer ii) to compare outcomes for people who had and had not recovered their QOL at 5 years.

\section{Methods}

The primary outcome was the Quality of Life in Adult Cancer Survivors Scale (QLACS). For each individual, the QLACS generic summary score (QLACS-GSS) at each time point was compared to the baseline score and classified as follows: improved $(>10 \%$ better), same (within 10\%) and worse ( $>10 \%$ deterioration). Exploratory univariate analyses were used to identify which variables were significantly different between the worse group and the combined same/improved groups at 5 years. For participants with a completed QLACS for at least 4 follow up time points, the pattern of movement between the three categories was explored.

\section{Results}

At 3 months, over $40 \%$ of people had worse QOL than at baseline (Figure 2). The proportion in the same group changed little over the five years. The number of people in the worse group gradually declined to 15 months and then levelled out at around a third, whilst the proportion in the improved group gradually increased to 2 years and then levelled out at $38 \%$. At five years, people in the worse group were significantly $(\mathrm{p}<0.05)$ more likely to have:

$>$ clinical levels of depression \& anxiety

$>$ low confidence to manage (self-efficacy)

$>$ worse health status \& worse global health/QOL

$>$ poor physical functioning

fatigue, pain, dyspnoea, \& bloating

Figure 3: How participants moved category

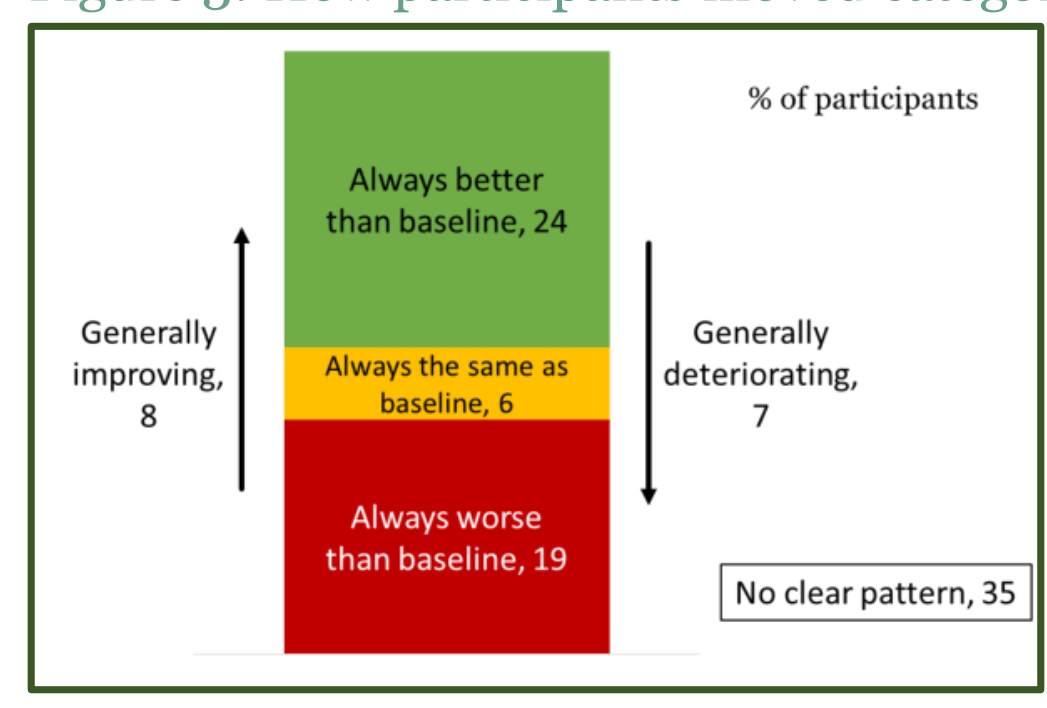

About half of participants did not change category (improved, same or worse) over follow up (Figure 3). A small number of patients generally improved over the five years, and a similar, small number generally deteriorated. later.

\section{Background}

\section{patients undergoing curative intent surgery.}

It systematically followed a cohort of patients from diagnosis using a framework of recovery over time (see Figure 1). participants (14\% of sample) gave a reduced level of consent to be followed up but to

suggested there was no selection bias.

Participants who continued to return questionnaires in the CREW study were broadly years were more likely to have been lost to follow up at 5 years.

- Questionnaires were administered pre-surgery and 3, 9, 15, 24, 36, 48 and 60 months

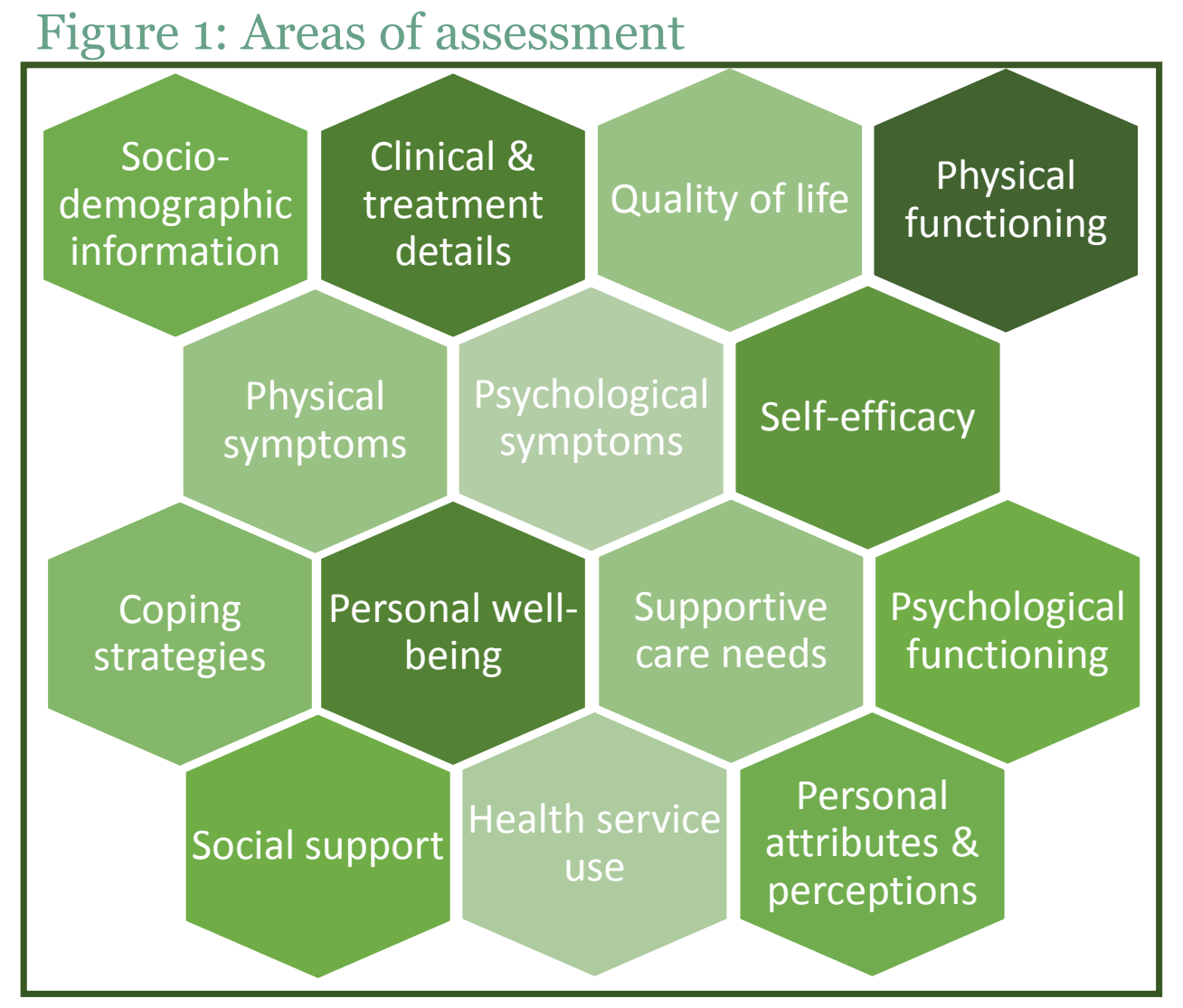

Figure 2: Changes in QLACS-GSS compared to baseline

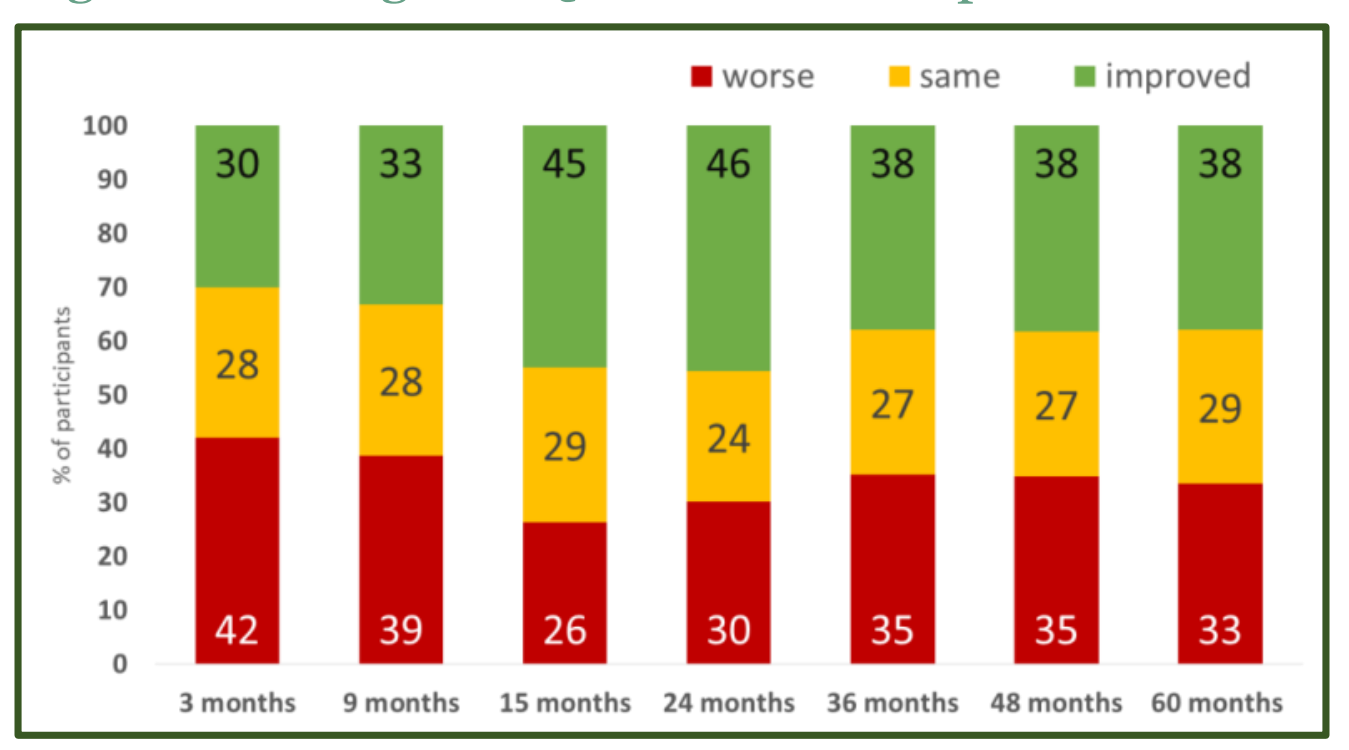

\section{Conclusions}

Whilst most people recover well from CRC, in terms of their health and well-being, about a third do not. Early psychosocial interventions, potentially pre-treatment, are needed to improve long term outcomes for CRC survivors.

\footnotetext{
References

Jansen, L., L. Koch, H. Brenner and V. A

England: 1990) 46(16): 2879-2888.

Fenlon, D., A. Richardson, J. Addington-Hall, P. Smith, J. Corner, J. Winter and C. Foster (2012). "A cohort study of the recovery of health and wellbeing following colorectal cancer

(CREW study): protocol paper." BMC Health Serv Res 12: 90.

Foster, C. and D. Fenlon (2011). "Recovery and self-management support following primary cancer treatment." Br J Cancer 105(S1): S21-S28.
} 